\title{
Availability and utilization of pigments from microalgae
}

\begin{abstract}
Microalgae are the major photosynthesizers on earth and produce important pigments that include chlorophyll a, b and c, $\beta$-carotene, astaxanthin, xanthophylls, and phycobiliproteins. Presently, synthetic colorants are used in food, cosmetic, nutraceutical, and pharmaceutical industries. However, due to problems associated with the harmful effects of synthetic colorants, exploitation of microalgal pigments as a source of natural colors becomes an attractive option. There are various factors such as nutrient availability, salinity, $\mathrm{pH}$, temperature, light wavelength, and light intensity that affect pigment production in microalgae. This paper reviews the availability and characteristics of microalgal pigments, factors affecting pigment production, and the application of pigments produced from microalgae. The potential of microalgal pigments as a source of natural colors is enormous as an alternative to synthetic coloring agents, which has limited applications due to regulatory practice for health reasons.
\end{abstract}

Keyword: Microalgae; Astaxanthin; Carotenoid; Chlorophyll; Phycobiliproteins; Pigments 